\title{
Dark matter annihilation through a lepton-specific Higgs boson
}

\author{
Heather E. Logan* \\ Ottawa-Carleton Institute for Physics, \\ Carleton University, Ottawa K1S 5B6 Canada
}

(Dated: November 1, 2010)

\begin{abstract}
It was recently argued by Hooper and Goodenough [1] that the excess gamma ray emission from within $1-2^{\circ}$ of the galactic center can be well-described by annihilation of $\sim 8 \mathrm{GeV}$ dark matter particles into tau pairs. I show that such a dark matter signal can be obtained naturally in the lepton-specific two-Higgs-doublet model extended by a stable singlet scalar dark matter candidate. The favored parameter region prefers a light Higgs state (below $200 \mathrm{GeV}$ ) with enhanced couplings to leptons and sizable invisible branching fraction. Part of the favored region leads to invisible decays of both of the CP-even neutral Higgs states.
\end{abstract}

\footnotetext{
* logan@physics.carleton.ca
} 


\section{INTRODUCTION}

Measurements of cosmic large-scale structure, galaxy and galactic-cluster dynamics, and gravitational lensing overwhelmingly support the existence of a non-baryonic, electrically neutral ("dark") component of the matter density of the universe. The observed dark matter density is consistent with its thermal production in the early universe if its mass and annihilation cross section to Standard Model (SM) particles are around the electroweak scale. The search for this weak-scale dark matter by means other than gravitational makes up a sizable fraction of the global experimental particle physics program.

Recently, Hooper and Goodenough [1] presented an independent analysis of gamma ray data from within $10^{\circ}$ of the galactic center collected over the past two years by the Fermi Gamma Ray Space Telescope. They showed that the spatial distribution and energy spectrum of gamma rays between about $1.25^{\circ}$ and $10^{\circ}$ of the galactic center are well described by a fit to a galactic disk profile and a spherically symmetric galactic bulge, with gamma rays originating from decays of neutral pions (produced in hadronic cosmic ray interactions) and inverse Compton scattering together with identified point sources.

This fit fails when extrapolated inward below $1.25^{\circ}$. Hooper and Goodenough identified a new component, highly peaked at the galactic center but not point-like 1 They argued that an astrophysical origin for this new component is implausible, and that instead the energy spectrum is very well fitted by annihilation of dark matter particles of mass $7.3-9.2 \mathrm{GeV}$ into $\tau$ pairs. Up to $10-20 \%$ of annihilations can be into $b \bar{b}$ or $c \bar{c}$ (which yield softer gammas) without degrading the fit. They further fit the spatial distribution of the new component assuming annihilation (i.e., assuming a rate proportional to the square of the number density) and find an inner density profile of $\rho \sim r^{-1.34 \pm 0.04}$. Assuming that this profile continues out to the distance of the sun and normalizing to the local dark matter density, the fitted rate implies a dark matter annihilation cross section of $\left\langle\sigma v_{\text {rel }}\right\rangle \simeq 3.3 \times 10^{-27} \mathrm{~cm}^{3} / \mathrm{s}$. Assuming instead that the profile is more peaked at the center of the galaxy due to attraction of the dark matter by the very high baryonic matter density there, and that the density profile softens to $\rho \sim r^{-1}$ outside the galactic core, they find an annihilation cross section of $1.5 \times 10^{-26} \mathrm{~cm}^{3} / \mathrm{s}$. These values are in rough agreement with the early universe cross section

${ }^{1}$ After removing this new component, the spectrum of the point source at the galactic center was extracted and is consistent with the extrapolation of the power law spectrum observed by ground-based gamma ray telescopes. 
required to obtain a thermal relic with the measured abundance, $\left\langle\sigma v_{\text {rel }}\right\rangle \simeq 3 \times 10^{-26} \mathrm{~cm}^{3} / \mathrm{s}$. The moderate discrepancy could be accounted for if the annihilation cross section is velocity dependent (such as for $P$-wave annihilations) or if there are additional annihilation modes, e.g., to electrons, muons, or neutrinos, which do not contribute significantly to the gamma ray signal.

A particularly simple model for dark matter is a stable, gauge-singlet, real scalar particle that annihilates through couplings to the Higgs sector [2]. Such a model was described recently in the current context in Ref. [3], in which a (complex) singlet scalar dark matter particle with mass of 10-30 GeV annihilates through s-channel SM Higgs exchange to SM fermions. Because ratios of the SM Higgs couplings to fermions are fixed by the fermion masses, annihilations into $b \bar{b}$ final states dominate for dark matter particle masses from just above the $b$ threshold up to about $65 \mathrm{GeV}$ where annihilations to off-shell $W$ bosons begin to turn on. In particular, a dominant annihilation mode to $\tau$ pairs can only be achieved if the couplings of the relevant exchanged particles can be made to favor the leptonic final state.

With this motivation I consider the annihilation of a gauge-singlet scalar dark matter candidate through interactions with the "lepton-specific" two-Higgs-doublet model (2HDML), in which one doublet generates masses for the SM quarks while the other generates masses for the leptons. This model has previously been considered in the context of dark matter annihilations in Ref. [4], which studied a variety of dark matter extensions of the 2HDM$\mathrm{L}$ with several-hundred-GeV dark matter particles annihilating or decaying into 2HDM-L states in order to explain the PAMELA and ATIC high-energy positron and electron excesses. Here I focus on annihilation of $\sim 8 \mathrm{GeV}$ dark matter particles through $s$-channel exchange of the two CP-even Higgs bosons of the 2HDM-L. I show that an appropriate annihilation cross section into $\tau$ pairs and sufficient suppression of hadronic final states can be achieved through appropriate choice of the model parameters. The dark matter annihilation cross section fitted in Ref. [1] favors a mass for the "leptonic" Higgs below 200-300 GeV. I also show that part of the preferred parameter region leads to large invisible decay branching fractions for both of the CP-even neutral Higgs bosons. Even though the dark matter particle receives contributions to its mass through couplings to the two Higgs doublets, its light mass can be obtained without too much fine tuning through appropriate choices for the couplings and vacuum expectation values of the two doublets. 
This paper is organized as follows. In Sec. II I present the model and summarize the relevant couplings. In Sec. III I compute the dark matter annihilation cross sections to $\tau$ pairs and quark pairs and determine the parameters necessary to agree with the fitted annihilation cross section of Ref. [1]. In Sec. IV I consider the question of naturalness of the small mass of the dark matter candidate. In Sec. $\mathrm{V}$ I compute the invisible Higgs decay branching fractions and comment on discovery prospects in various parts of parameter space. In Sec. VI I comment on the spin-independent dark matter direct detection cross section in this scenario. Conclusions are summarized in Sec. VII.

\section{THE MODEL}

The lepton-specific two-Higgs-doublet model contains two complex SU(2)-doublet scalar fields, $\Phi_{q}$ and $\Phi_{\ell}$, where $\Phi_{q}$ generates the masses of both up- and down-type quarks while $\Phi_{\ell}$ generates the masses of the charged leptons. This Yukawa coupling structure was first introduced in Refs. [5-7] and the couplings, decays, and phenomenology of the resulting Higgs states have been studied in Refs. [4, 8-14]. This Yukawa structure was also used in the neutrino mass model of Ref. [15].

The two doublets can be written as,

$$
\Phi_{i}=\left(\begin{array}{c}
\phi_{i}^{+} \\
\frac{1}{\sqrt{2}}\left(\phi_{i}^{0, r}+v_{i}+i \phi_{i}^{0, i}\right)
\end{array}\right), \quad i=q, \ell
$$

The vacuum expectation values are constrained by $v_{q}^{2}+v_{\ell}^{2}=v_{\mathrm{SM}}^{2}=(246 \mathrm{GeV})^{2}$, with their ratio parameterized as

$$
\tan \beta \equiv \frac{v_{q}}{v_{\ell}}
$$

The desired form of the Yukawa Lagrangian is enforced by imposing a $Z_{2}$ symmetry, broken only softly in the Higgs potential, under which $\Phi_{\ell}$ and the right-handed leptons transform as $\Phi_{\ell} \rightarrow-\Phi_{\ell}$ and $e_{R i} \rightarrow-e_{R i}$, with all other fields unaffected. The Yukawa Lagrangian is then,

$$
\mathcal{L}_{\text {Yuk }}=-\sum_{i, j=1}^{3}\left[y_{i j}^{u} \bar{u}_{R i} \widetilde{\Phi}_{q}^{\dagger} Q_{L j}+y_{i j}^{d} \bar{d}_{R i} \Phi_{q}^{\dagger} Q_{L j}+y_{i j}^{\ell} \bar{e}_{R i} \Phi_{\ell}^{\dagger} L_{L j}\right]+\text { h.c. },
$$

where $i, j$ are generation indices, $y_{i j}^{u, d, \ell}$ are the Yukawa coupling matrices, the left-handed 
quark and lepton doublets are given by $Q_{L i}=\left(u_{L i}, d_{L i}\right)^{T}$ and $L_{L i}=\left(\nu_{L i}, e_{L i}\right)^{T}$, and the conjugate Higgs doublet is,

$$
\widetilde{\Phi}_{q} \equiv i \sigma_{2} \Phi_{q}^{*}=\left(\begin{array}{c}
\frac{1}{\sqrt{2}}\left(\phi_{q}^{0, r}+v_{q}-i \phi_{q}^{0, i}\right) \\
-\phi_{q}^{-}
\end{array}\right)
$$

The scalar potential for $\Phi_{q, \ell}$ was given, e.g., in Eq. (2) of Ref. [12].

To this model I add a gauge-singlet real scalar field $S$, which is odd under a second, unbroken global $Z_{2}$ symmetry. This second $Z_{2}$ ensures that $S$ is stable. The Higgs potential acquires the following new terms:

$$
V_{S}=\frac{1}{2} m_{3}^{2} S^{2}+\frac{\lambda_{q}}{2} S^{2} \Phi_{q}^{\dagger} \Phi_{q}+\frac{\lambda_{\ell}}{2} S^{2} \Phi_{\ell}^{\dagger} \Phi_{\ell}+\lambda_{S} S^{4}
$$

Note that a term $\sim S^{2} \Phi_{q}^{\dagger} \Phi_{\ell}$ is forbidden by the requirement that the $Z_{2}$ responsible for the form of the Yukawa Lagrangian is only softly broken.

After electroweak symmetry breaking, the couplings $\lambda_{q, \ell}$ lead to three-point couplings of two $S$ particles to the CP-even neutral states in $\Phi_{q, \ell}$. We parameterize the CP-even neutral mass eigenstates in the usual way as

$$
\begin{aligned}
h^{0} & =-\sin \alpha \phi_{\ell}^{0, r}+\cos \alpha \phi_{q}^{0, r}, \\
H^{0} & =\cos \alpha \phi_{\ell}^{0, r}+\sin \alpha \phi_{q}^{0, r} .
\end{aligned}
$$

The Feynman rules for the couplings of these states to quarks, leptons, $W$ and $Z$ bosons, and $S$ pairs are then,

$$
\begin{aligned}
& h^{0} q \bar{q}:-i \frac{m_{q}}{v_{\mathrm{SM}}} \frac{\cos \alpha}{\sin \beta}, \quad \quad h^{0} \ell \bar{\ell}: i \frac{m_{\ell}}{v_{\mathrm{SM}}} \frac{\sin \alpha}{\cos \beta}, \quad \quad h^{0} V_{\mu} V_{\nu}: 2 i \frac{M_{V}^{2}}{v_{\mathrm{SM}}} \sin (\beta-\alpha) g_{\mu \nu}, \\
& h^{0} S S:-i v_{\mathrm{SM}}\left(\lambda_{q} \sin \beta \cos \alpha-\lambda_{\ell} \cos \beta \sin \alpha\right) \text {, } \\
& H^{0} q \bar{q}:-i \frac{m_{q}}{v_{\mathrm{SM}}} \frac{\sin \alpha}{\sin \beta}, \quad \quad H^{0} \ell \bar{\ell}:-i \frac{m_{\ell}}{v_{\mathrm{SM}}} \frac{\cos \alpha}{\cos \beta}, \quad \quad H^{0} V_{\mu} V_{\nu}: 2 i \frac{M_{V}^{2}}{v_{\mathrm{SM}}} \cos (\beta-\alpha) g_{\mu \nu} \text {, } \\
& H^{0} S S:-i v_{\mathrm{SM}}\left(\lambda_{q} \sin \beta \sin \alpha+\lambda_{\ell} \cos \beta \cos \alpha\right) \text {. }
\end{aligned}
$$

In what follows I will consider as an example the limit $\cos (\beta-\alpha)=0$, i.e., $\cos \alpha \rightarrow \sin \beta$ 
and $\sin \alpha \rightarrow-\cos \beta$. In this limit the couplings become,

$$
\begin{aligned}
& h^{0} q \bar{q}:-i \frac{m_{q}}{v_{\mathrm{SM}}}, \quad h^{0} \ell \bar{\ell}:-i \frac{m_{\ell}}{v_{\mathrm{SM}}}, \quad h^{0} V_{\mu} V_{\nu}: 2 i \frac{M_{V}^{2}}{v_{\mathrm{SM}}} g_{\mu \nu}, \\
& h^{0} S S:-i v_{\mathrm{SM}}\left(\lambda_{q} \sin ^{2} \beta+\lambda_{\ell} \cos ^{2} \beta\right), \\
& H^{0} q \bar{q}: i \frac{m_{q}}{v_{\mathrm{SM}}} \cot \beta, \\
& H^{0} \ell \bar{\ell}:-i \frac{m_{\ell}}{v_{\mathrm{SM}}} \tan \beta,
\end{aligned}
$$

\section{DARK MATTER ANNIHILATION SIGNATURE}

In this model, the annihilation of two $S$ particles proceeds via $s$-channel exchange of $h^{0}$ and $H^{0}$. Neglecting the kinetic energies of the nonrelativistic initial-state dark matter particles, the annihilation cross section into fermions $f \bar{f}$ is given by,

$$
\sigma v_{\mathrm{rel}}=\frac{N_{c} m_{f}^{2}}{4 \pi} C_{f}^{2}\left[1-\frac{4 m_{f}^{2}}{s}\right]^{3 / 2}
$$

where $v_{\text {rel }}$ is the relative velocity of the two $S$ particles, $N_{c}$ is the number of colors of fermion

species $f, s$ is the square of the center-of-mass energy (equal to $4 M_{S}^{2}$ in the nonrelativistic limit), and $C_{f}$ is the relevant product of coupling and propagator factors given for leptons and quarks by,

$$
\begin{aligned}
C_{\ell} & =\frac{\left(\lambda_{q} \tan \beta \cos \alpha-\lambda_{\ell} \sin \alpha\right) \sin \alpha}{s-M_{h}^{2}}-\frac{\left(\lambda_{q} \tan \beta \sin \alpha+\lambda_{\ell} \cos \alpha\right) \cos \alpha}{s-M_{H}^{2}} \\
C_{q} & =-\frac{\left(\lambda_{q} \cos \alpha-\lambda_{\ell} \cot \beta \sin \alpha\right) \cos \alpha}{s-M_{h}^{2}}-\frac{\left(\lambda_{q} \sin \alpha+\lambda_{\ell} \cot \beta \cos \alpha\right) \sin \alpha}{s-M_{H}^{2}} .
\end{aligned}
$$

Here $M_{h}$ and $M_{H}$ are the masses of $h^{0}$ and $H^{0}$, respectively. Note that averaging over the dark matter particle velocity distribution has no effect on this cross section because the cross section is velocity-independent in the low-velocity limit.

Taking $M_{S}=8 \mathrm{GeV}$, the cross section for $S S \rightarrow \tau \tau$ yields the constraint

$$
C_{\ell}^{2}=\left(\frac{1}{128 \mathrm{GeV}}\right)^{4}\left[\frac{\sigma v_{\mathrm{rel}}}{10^{-26} \mathrm{~cm}^{3} / \mathrm{s}}\right]
$$

Recall that the fit in Ref. [1] found an annihilation cross section to $\tau \tau$ in the range $3.3 \times 10^{-27}$ to $1.5 \times 10^{-26} \mathrm{~cm}^{3} / \mathrm{s}$. 
The fit in Ref. [1] requires that $b \bar{b}$ and $c \bar{c}$ final states make up no more than $10-20 \%$ of annihilations, i.e.,

$$
\frac{\sigma v_{\mathrm{rel}}(S S \rightarrow b \bar{b}+c \bar{c})}{\sigma v_{\mathrm{rel}}(S S \rightarrow \tau \tau)} \lesssim 0.2
$$

If the coupling factors $C_{\ell}$ and $C_{q}$ were equal, the ratio of annihilation cross sections given above would be equal to the ratio of the corresponding decay widths of a SM-like Higgs boson of mass $2 M_{S}$. Taking $M_{S}=8 \mathrm{GeV}$ (the relevant SM Higgs branching ratios do not vary much over the favored dark matter mass range of 7.3-9.2 GeV), I find using the public code HDECAY version 3.53 [16],

$$
\frac{\Gamma\left(H_{\mathrm{SM}} \rightarrow b \bar{b}+c \bar{c}\right)}{\Gamma\left(H_{\mathrm{SM}} \rightarrow \tau \tau\right)}=13.3
$$

This yields an upper bound on the ratio of coupling factors,

$$
\frac{C_{q}^{2}}{C_{\ell}^{2}} \lesssim \frac{0.2}{13.3}=0.015, \quad \text { or } \quad\left|\frac{C_{q}}{C_{\ell}}\right| \lesssim 0.12
$$

implying that $C_{q}$ must be suppressed by about an order of magnitude relative to $C_{\ell}$. To characterize the parameter region in which the excess gamma ray signature can be accommodated, I examine $C_{\ell}$ and $C_{q}$ in two limits.

First, consider the case when $\phi_{\ell}^{0, r}$ and $\phi_{q}^{0, r}$ are mass eigenstates.2 Then the coupling factors become,

$$
C_{\ell}=\frac{\lambda_{\ell}}{M_{\phi_{\ell}^{0, r}}^{2}-s}, \quad C_{q}=\frac{\lambda_{q}}{M_{\phi_{q}^{0, r}}^{2}-s} .
$$

In particular, $S S \rightarrow \ell \bar{\ell}$ proceeds only through $\phi_{\ell}^{0, r}$ exchange with $S S$ coupling $\lambda_{\ell}$, and $S S \rightarrow q \bar{q}$ proceeds only through $\phi_{q}^{0, r}$ exchange with $S S$ coupling $\lambda_{q}$. Taking $\lambda_{q}$ small is enough to suppress the $q \bar{q}$ final states.

The required value of $C_{\ell}$ from Eq. (11) yields a relation between $\lambda_{\ell}$ and the $\phi_{\ell}^{0, r}$ mass; neglecting $s=(16 \mathrm{GeV})^{2}$ relative to $M_{\phi_{\ell}^{0, r}}^{2}$, I find,

$$
\lambda_{\ell} \simeq\left[\frac{M_{\phi_{\ell}^{0, r}}}{128 \mathrm{GeV}}\right]^{2}\left[\frac{\sigma v_{\mathrm{rel}}}{10^{-26} \mathrm{~cm}^{3} / \mathrm{s}}\right]^{1 / 2} .
$$

In particular, the required dark matter annihilation cross section is naturally obtained with

\footnotetext{
${ }^{2}$ This basis can be automatically chosen if $h^{0}$ and $H^{0}$ are degenerate.
} 
$\lambda_{\ell} \sim 1$ and the $\phi_{\ell}^{0, r}$ mass around $130 \mathrm{GeV}$. For other masses, the required coupling $\lambda_{\ell}$ scales proportional to the square of the $\phi_{\ell}^{0, r}$ mass. The upper bound on hadronic final states from Eq. (14) leads to the constraint,

$$
\lambda_{q} \lesssim 0.12 \frac{M_{\phi_{q}^{0, r}}^{2}}{M_{\phi_{\ell}^{0, r}}^{2}} \lambda_{\ell} .
$$

Taking $\lambda_{\ell}$ and $M_{\phi_{\ell}^{0, r}}$ as in Eq. (16), the required suppression can be achieved either by making $\lambda_{q}$ small or by making $\phi_{q}^{0, r}$ heavy; $\lambda_{q} \sim 1$ requires $M_{\phi_{q}^{0, r}} \gtrsim 370 \mathrm{GeV}$.

Second, consider the case of $\cos (\beta-\alpha) \rightarrow 0$. In this limit, the couplings of $h^{0}$ to SM particles are the same as those of the SM Higgs and the $H^{0} W W$ and $H^{0} Z Z$ couplings are zero [see Eq. (8)]. The coupling and propagator factors become,

$$
\begin{aligned}
& C_{\ell}=\frac{\lambda_{q} \sin ^{2} \beta+\lambda_{\ell} \cos ^{2} \beta}{M_{h}^{2}-s}+\frac{\left(\lambda_{\ell}-\lambda_{q}\right) \sin ^{2} \beta}{M_{H}^{2}-s} \\
& C_{q}=\frac{\lambda_{q} \sin ^{2} \beta+\lambda_{\ell} \cos ^{2} \beta}{M_{h}^{2}-s}-\frac{\left(\lambda_{\ell}-\lambda_{q}\right) \cos ^{2} \beta}{M_{H}^{2}-s} .
\end{aligned}
$$

In this case, taking $\lambda_{q}$ small is not enough to suppress the $q \bar{q}$ final states. One also needs the quantity

$$
\lambda_{\ell} \cos ^{2} \beta\left[\frac{1}{M_{h}^{2}-s}-\frac{1}{M_{H}^{2}-s}\right]
$$

to be small, without making $\lambda_{\ell}$ small. This can be achieved by taking $M_{H} \rightarrow M_{h}$ or by taking $\tan \beta$ large $\left(\tan \beta \gtrsim 3 \sqrt{\lambda_{\ell}}\right.$ is sufficient). Large $\tan \beta$ corresponds to making $H^{0}$ "lepton-friendly," as can be seen from the couplings to fermions given in Eq. (8)). In the large $\tan \beta$ limit I obtain,

$$
C_{\ell} \simeq \frac{\lambda_{q}}{M_{h}^{2}-s}+\frac{\lambda_{\ell}-\lambda_{q}}{M_{H}^{2}-s}, \quad C_{q} \simeq \frac{\lambda_{q}}{M_{h}^{2}-s} .
$$

In this limit, the $S S \rightarrow q \bar{q}$ channel is suppressed by keeping $\lambda_{q}$ small; then the $S S \rightarrow \tau \tau$ cross section is due primarily to exchange of $H^{0}$ through the $\lambda_{\ell}$ coupling.

For large $\tan \beta$, the upper bound on hadronic final states from Eq. (14) yields the rough constraint (neglecting $s$ relative to the squared Higgs masses and neglecting $\lambda_{q}$ relative to $\lambda_{\ell}$ in the second term of $C_{\ell}$ ),

$$
\lambda_{q} \lesssim 0.14 \frac{M_{h}^{2}}{M_{H}^{2}} \lambda_{\ell}
$$


Then, neglecting the $\lambda_{q}$ contributions to $C_{\ell}$, I again obtain from Eq. (11),

$$
\lambda_{\ell} \simeq\left[\frac{M_{H}}{128 \mathrm{GeV}}\right]^{2}\left[\frac{\sigma v_{\mathrm{rel}}}{10^{-26} \mathrm{~cm}^{3} / \mathrm{s}}\right]^{1 / 2}
$$

In particular, taking $\sigma v_{\text {rel }}=10^{-26} \mathrm{~cm}^{3} / \mathrm{s}$ and a heavier second Higgs state $H^{0}$ of 200 (300) $\mathrm{GeV}$ would require an enhanced coupling $\lambda_{\ell} \simeq 2.4$ (5.5). If the dark matter annihilation cross section is near the lower end of the fitted range of Ref. [1], $\sigma v_{\text {rel }} \simeq 3.3 \times 10^{-27} \mathrm{~cm}^{3} / \mathrm{s}$, this is reduced to $\lambda_{\ell} \simeq 1.4(3.2)$. However, this low end of the annihilation cross section range is disfavored in the model discussed here because there is no way to boost the total annihilation cross section in the early universe up to the range favored by the observed dark matter relic density. Depending on the model, such a boost can be achieved in a number of ways: through an annihilation cross section that grows with velocity as for $P$-wave annihilations, through co-annihilations in the early universe with another particle carrying the dark matter parity, or through the presence of additional annihilation final states that do not contribute significantly to the gamma-ray flux and thus are not included in the annihilation cross section fitted from gamma-ray data. None of these mechanisms apply in the model considered here. Alternatively, the fitted annihilation cross section could be increased if the transition to the steeper density profile were assumed to happen closer to the galactic center; this would in turn favor an even lighter $H^{0}$ for a given $\lambda_{\ell}$. Therefore, avoiding a large quartic scalar coupling strongly favors a light lepton-friendly Higgs.

\section{NATURALNESS OF THE DARK MATTER PARTICLE MASS}

Reference [1] found that the gamma ray excess around the galactic center is best fitted with a dark matter particle mass of 7.3-9.2 GeV. The scalar potential in Eq. (5) leads to a mass for the scalar $S$ in terms of the underlying model parameters of

$$
M_{S}^{2}=m_{3}^{2}+\frac{v_{\mathrm{SM}}^{2}}{2}\left(\lambda_{q} \sin ^{2} \beta+\lambda_{\ell} \cos ^{2} \beta\right) .
$$

We have seen that the coupling $\lambda_{\ell}$ must be at least $\mathcal{O}(1)$ to accommodate the fitted annihilation cross section, while $\lambda_{q}$ should be at least $\sim 10$ times smaller to avoid too large an annihilation cross section to hadronic final states. 
Large cancellations in Eq. (23) can be avoided if $\tan \beta$ is moderately large; $\tan \beta \sim 17$ results in a mass-squared contribution from $v_{\mathrm{SM}}^{2} \lambda_{\ell} \cos ^{2} \beta / 2$ of about $(10 \mathrm{GeV})^{2}$. Avoiding fine tuning between the remaining terms requires $\lambda_{q}$ to be significantly smaller than the constraint from hadronic annihilation final states; a mass-squared contribution from $v_{\mathrm{SM}}^{2} \lambda_{q} \sin ^{2} \beta / 2$ of about $(10 \mathrm{GeV})^{2}$ requires $\lambda_{q} \sim 1 / 300$.

The mass term $m_{3}^{2}$ suffers of course from the same quadratic sensitivity to high energy scales that leads to the hierarchy problem for the SM Higgs mass. In this case, however, the quadratically divergent one-loop contribution to the renormalized mass-squared comes solely from loops of $\Phi_{\ell}$ via the coupling $\lambda_{\ell}$ (the $\Phi_{q}$ contribution is suppressed by the small coupling $\lambda_{q}$; likewise, loops of $S$ can be suppressed by making $\lambda_{S}$ small in Eq. (5-5)), and is given by

$$
\delta M_{S}^{2}=\frac{\lambda_{\ell}}{4 \pi^{2}} \Lambda^{2}
$$

where $\Lambda$ is the cutoff. While naturalness of the SM Higgs mass-squared parameter with no more than, say, $10 \%$ fine tuning requires cancellation of the top quark loop quadratic

divergence around $2 \mathrm{TeV}$ (for a review, see, e.g., Ref. [17]), naturalness at this level for $M_{S}^{2} \simeq(10 \mathrm{GeV})^{2}$ requires that the $\Phi_{\ell}$ loop quadratic divergence be cancelled around $200 \mathrm{GeV}$ (by, e.g., supersymmetric partners of the $\Phi_{\ell}$ states - Higgsinos around this mass scale are a common feature of supersymmetric models).

\section{CONSEQUENCES FOR HIGGS DECAYS}

The presence of a stable singlet $S$ with mass around $8 \mathrm{GeV}$ and couplings to $h^{0}$ and $H^{0}$ has profound implications for Higgs collider signatures. The partial width for $\phi \rightarrow S S$, with $\phi=h^{0}$ or $H^{0}$, is given by

$$
\Gamma(\phi \rightarrow S S)=\frac{\lambda_{\phi}^{2} v_{\mathrm{SM}}^{2}}{16 \pi M_{\phi}} \sqrt{1-\frac{4 M_{S}^{2}}{M_{\phi}^{2}}}
$$

where the couplings for $h^{0}$ and $H^{0}$ are given by

$$
\begin{aligned}
& \lambda_{h}=\lambda_{q} \sin \beta \cos \alpha-\lambda_{\ell} \cos \beta \sin \alpha, \\
& \lambda_{H}=\lambda_{q} \sin \beta \sin \alpha+\lambda_{\ell} \cos \beta \cos \alpha .
\end{aligned}
$$


I again focus on the case $\cos (\beta-\alpha) \rightarrow 0$. The couplings of $h^{0}$ and $H^{0}$ to scalars become,

$$
\begin{aligned}
\lambda_{h} & =\lambda_{q} \sin ^{2} \beta+\lambda_{\ell} \cos ^{2} \beta, \\
\lambda_{H} & =\left(\lambda_{\ell}-\lambda_{q}\right) \sin \beta \cos \beta .
\end{aligned}
$$

I first consider the SM-like Higgs $h^{0}$. The coupling $\lambda_{h}$ enters $C_{q}$ (see Eq. (18) above), so that the constraint on hadronic annihilations leads very roughly to $\lambda_{h} \lesssim 0.1 \lambda_{\ell}$. This limit is saturated for $\lambda_{\ell} \sim 1, \lambda_{q} \sim 0.1$, and $\tan \beta \sim 3$. Neglecting the kinematic factor $\sqrt{1-4 M_{S}^{2} / M_{h}^{2}}$, the invisible width of $h^{0}$ is given by,

$$
\Gamma\left(h^{0} \rightarrow S S\right) \simeq 99 \mathrm{MeV}\left[\frac{\lambda_{h}}{0.1}\right]^{2}\left[\frac{120 \mathrm{GeV}}{M_{h}}\right]
$$

When $\cos (\beta-\alpha)=0$, the couplings of $h^{0}$ to SM particles all become equal to their SM values. The total width of the SM Higgs, corresponding to the visible width of $h^{0}$, is then 3.6 (8.3, 82, 630) $\mathrm{MeV}$ for $M_{h}=120(140,160,180) \mathrm{GeV}$ [16]. For $\lambda_{h} \sim 0.1$, then, invisible decays to $S S$ dominate the total width of $h^{0}$ for masses below the $W W$ threshold. Above the $W W$ threshold, the invisible branching fraction falls quickly (to about $10 \%$ at $M_{h}=180 \mathrm{GeV}$ ) due to the rapid growth of the Higgs decay widths to vector bosons. If instead I take $\lambda_{q}$ and $\tan \beta$ values as dictated by full naturalness of $M_{S}$, i.e., requiring $v_{\mathrm{SM}}^{2} \lambda_{h} / 2 \sim(10 \mathrm{GeV})^{2}$, I obtain $\lambda_{h} \sim 1 / 300$. This yields an invisible width for $h^{0}$ around $0.1 \mathrm{MeV}$, leading to an invisible branching fraction below $3 \%$ even for $h^{0}$ masses below the $W W$ threshold. Allowing $10 \%$ fine tuning for the $S$ mass yields $\lambda_{h} \sim 1 / 30$ and an invisible width for $h^{0}$ around $10 \mathrm{MeV}$, leading to an invisible branching fraction of about 75\% (50\%) for $M_{h}=120$ (140) GeV. A recent ATLAS study [18] of an invisibly-decaying Higgs produced in vector boson fusion or associated production with a $Z$ boson finds a $95 \%$ confidence level exclusion reach with $30 \mathrm{fb}^{-1}$ down to invisible branching fractions of about $50 \%$ in this mass range, assuming SM production cross sections.

Now consider the second Higgs boson $H^{0}$. In order to avoid needing too large a coupling $\lambda_{\ell}$, I will assume that $M_{H}$ is below the $t \bar{t}$ threshold, $M_{H} \lesssim 350 \mathrm{GeV}$. Then the partial width of $H^{0}$ to SM particles is dominated by $H^{0} \rightarrow \tau \tau$ for $\tan \beta \gtrsim 2$. The partial width for 
$H^{0} \rightarrow \tau \tau$ is given by,

$$
\Gamma\left(H^{0} \rightarrow \tau \tau\right)=\frac{M_{H} m_{\tau}^{2} \tan ^{2} \beta}{8 \pi v_{\mathrm{SM}}^{2}}\left[1-\frac{4 m_{\tau}^{2}}{M_{H}^{2}}\right]^{3 / 2} \simeq 0.27 \mathrm{MeV} \tan ^{2} \beta\left[\frac{M_{H}}{130 \mathrm{GeV}}\right]
$$

where in the last step I ignored the $\tau$ mass in the kinematics. The partial width for $H^{0} \rightarrow S S$ is given by

$$
\Gamma\left(H^{0} \rightarrow S S\right) \simeq 9.2 \mathrm{GeV} \lambda_{H}^{2}\left[\frac{130 \mathrm{GeV}}{M_{H}}\right]
$$

Neglecting $\lambda_{q}$, taking $\tan \beta \gtrsim 3$, and applying the fitted dark matter annihilation cross section from Eq. (22), the coupling $\lambda_{H}$ is approximately given by

$$
\lambda_{H} \simeq \cot \beta\left[\frac{M_{H}}{128 \mathrm{GeV}}\right]^{2}\left[\frac{\sigma v_{\mathrm{rel}}}{10^{-26} \mathrm{~cm}^{3} / \mathrm{s}}\right]^{1 / 2}
$$

Inserting this into the previous equation with $\sigma v_{\text {rel }}=10^{-26} \mathrm{~cm}^{3} / \mathrm{s}$, the partial width for $H^{0} \rightarrow S S$ becomes,

$$
\Gamma\left(H^{0} \rightarrow S S\right) \simeq 9.8 \mathrm{GeV} \cot ^{2} \beta\left[\frac{M_{H}}{130 \mathrm{GeV}}\right]^{3}
$$

Sufficient suppression of hadronic final states in $S S$ annihilation requires $\tan \beta \gtrsim 3$, while full naturalness of $M_{S}$ favors $\tan \beta \sim 17$. Taking $M_{H}=130 \mathrm{GeV}$ and $\tan \beta=3$ yields an overwhelmingly invisibly decaying $H^{0}$, with only about 2 per mille visible decays to $\tau \tau$. Taking $\tan \beta=17$ yields an invisible branching fraction for $H^{0}$ of about $30 \%$ for this mass, with the remainder of decays to $\tau \tau$. The ratio of invisible to visible widths grows with the $H^{0}$ mass like $M_{H}^{2}$, yielding an invisible branching fraction of about $50 \%$ at $M_{H}=200 \mathrm{GeV}$; this growth is due to the larger coupling $\lambda_{\ell}$ required to obtain the fitted dark matter annihilation cross section at higher $H^{0}$ masses. Allowing $10 \%$ fine tuning for the $S$ mass yields $\tan \beta \sim 5.5$ for $\lambda_{\ell} \sim 1$, leading to a visible width (to $\tau \tau$ ) of a $130 \mathrm{GeV} H^{0}$ of about $2.5 \%$, with the remainder of decays invisible.

Discovery of an invisibly-decaying $H^{0}$ at the LHC is a major challenge. When $\cos (\beta-$ $\alpha) \rightarrow 0, H^{0}$ is not produced in vector boson fusion or in association with a $W$ or $Z$ boson. Its cross sections in gluon fusion and $t \bar{t} H^{0}$ associated production are suppressed by $\cot ^{2} \beta$. Instead, its primary production modes are through $s$-channel $Z(W)$ exchange in association with a CP-odd (charged) Higgs, which decays predominantly to $\tau \tau(\tau \nu)$. Discovery and 
characterization of such a particle may have to wait for a high-energy $e^{+} e^{-}$collider. Because electroweak precision constraints tend to disfavor large mass splittings among the members of a second Higgs doublet, the International Linear Collider operating with a center-ofmass energy around $500 \mathrm{GeV}$ should be able to study this model in detail (for a review of International Linear Collider capabilities for the second Higgs doublet of the minimal supersymmetric standard model, see, e.g., Ref. [19]).

\section{DIRECT DETECTION CROSS SECTION}

Scalar dark matter particles $S$ in the galactic halo can scatter off nuclei via exchange of $h^{0}$ and $H^{0}$, yielding direct recoil signals for dark matter. In this process, $h^{0}$ and $H^{0}$ must couple to the quarks or gluons in the nucleus; the scattering cross section is thus suppressed by the same physics that yields the suppression of annihilations to hadronic final states. Following, e.g., Ref. [20], the spin-independent cross section for $S$ scattering off the proton is

$$
\sigma_{\mathrm{SI}}=\frac{m_{p}^{4}}{2 \pi\left(m_{p}+M_{S}\right)^{2}} C_{q}^{2}\left[f_{p u}+f_{p d}+f_{p s}+\frac{2}{27}\left(3 f_{G}\right)\right]^{2},
$$

where $m_{p}$ is the proton mass, $C_{q}$ is the coupling and propagator factor given in Eq. (10) evaluated at $s=0$, and the nuclear formfactors in the square brackets are given by [21],

$$
f_{p u}=0.02, \quad f_{p d}=0.026, \quad f_{p s}=0.118, \quad f_{G}=0.836
$$

with an uncertainty of about $30 \%$.

Neglecting $\left(2 M_{S}\right)^{2}$ compared to $M_{h}^{2}, M_{H}^{2}$ in the coupling and propagator factors, this can be expressed in terms of the parameter region favored by the annihilation signal according to,

$$
\sigma_{\mathrm{SI}} \simeq 4.0 \times 10^{-42} \mathrm{~cm}^{2}\left[\frac{9 \mathrm{GeV}}{m_{p}+M_{S}}\right]^{2}\left[\frac{\sigma v_{\mathrm{rel}}}{10^{-26} \mathrm{~cm}^{3} / \mathrm{s}}\right]\left[\frac{C_{q}^{2} / C_{\ell}^{2}}{0.015}\right],
$$

where the term in the last set of square brackets is at most equal to 1 due to the upper bound of $\sim 20 \%$ on the fraction of dark matter annihilations to hadrons. We thus obtain an upper bound on the direct detection cross section roughly an order of magnitude below the cross section required for the (controversial) dark matter interpretation of the CoGeNT

data [22]. This cross section is likewise about a factor of 5 below the current upper limit 
from the XENON100 experiment [23] for $M_{S}=8 \mathrm{GeV}$.

\section{CONCLUSIONS}

Hooper and Goodenough [1] identified an excess component of the gamma ray flux near the galactic center as measured by the Fermi Gamma Ray Space Telescope and showed that it is well fitted by annihilations of $\sim 8 \mathrm{GeV}$ dark matter particles into $\tau \tau$ final states. I showed in this paper that this scenario can be realized in the lepton-specific two-Higgsdoublet model extended by a stable gauge-singlet scalar dark matter particle.

Obtaining the right annihilation cross section requires $M / \sqrt{\lambda_{\ell}} \sim 130 \mathrm{GeV}$, where $M$ is the mass of the CP-even neutral Higgs state that lives mostly in the "lepton-friendly" Higgs doublet $\Phi_{\ell}$ and $\lambda_{\ell}$ is the four-point coupling of $\Phi_{\ell}$ to dark matter particle pairs. Avoiding too large a four-point coupling thus forces the lepton-friendly Higgs to be light. Suppressing annihilations to quarks requires both the four-point coupling of the "quark-friendly" doublet to dark matter and the mixing between the two doublets to be small. Complete naturalness of the rather low dark matter particle mass can be achieved if the vacuum expectation value of $\Phi_{\ell}$ is around $14 \mathrm{GeV}$ (i.e., $\tan \beta \sim 17$ ) and the four-point coupling of the quarkfriendly doublet to dark matter is around 1/300 or less - in itself a rather fine-tuned situation. Allowing $10 \%$ fine tuning for the $S$ mass leads to the more comfortable values of $\tan \beta \sim 5.5$ and $\lambda_{q} \lesssim 1 / 30$.

The most significant consequences of this scenario for collider physics are as follows. First, both of the CP-even neutral Higgs states should be in the 100-200 GeV mass range in order to avoid too large a coupling $\lambda_{\ell}$. Second, in part of the allowed parameter space, both of the CP-even neutral Higgs states will decay predominantly to dark matter particles; requiring no more than $10 \%$ fine tuning for the dark matter particle mass, the Standard Model-like Higgs acquires an invisible width comparable to its visible width, while the lepton-friendly Higgs decays mostly invisibly with a branching fraction to $\tau \tau$ of a few percent. Because of the suppression of their single-production couplings to quarks and gauge bosons, discovery of the four Higgs states of the lepton-friendly doublet in this scenario is a major challenge at the LHC, but should be straightforward at a $500 \mathrm{GeV}$ International Linear $e^{+} e^{-}$Collider. 


\section{ACKNOWLEDGMENTS}

I thank Thomas Gregoire for helpful discussions. This work was supported by the Natural Sciences and Engineering Research Council of Canada.

[1] D. Hooper and L. Goodenough, arXiv:1010.2752 [hep-ph].

[2] V. Silveira and A. Zee, Phys. Lett. B 161, 136 (1985); C. P. Burgess, M. Pospelov and T. ter Veldhuis, Nucl. Phys. B 619, 709 (2001) arXiv:hep-ph/0011335].

[3] V. Barger, Y. Gao, M. McCaskey and G. Shaughnessy, Phys. Rev. D 82, 095011 (2010) arXiv:1008.1796 [hep-ph]].

[4] H. S. Goh, L. J. Hall and P. Kumar, JHEP 0905, 097 (2009) arXiv:0902.0814 [hep-ph]].

[5] R. M. Barnett, G. Senjanovic, L. Wolfenstein and D. Wyler, Phys. Lett. B 136, 191 (1984).

[6] R. M. Barnett, G. Senjanovic and D. Wyler, Phys. Rev. D 30, 1529 (1984).

[7] Y. Grossman, Nucl. Phys. B 426, 355 (1994) arXiv:hep-ph/9401311.

[8] A. G. Akeroyd and W. J. Stirling, Nucl. Phys. B 447, 3 (1995); A. G. Akeroyd, Phys. Lett. B 377, 95 (1996) arXiv:hep-ph/9603445]; Nucl. Phys. B 544, 557 (1999) arXiv:hep-ph/9806337.

[9] A. G. Akeroyd, J. Phys. G 24, 1983 (1998) arXiv:hep-ph/9803324.

[10] V. Barger, H. E. Logan and G. Shaughnessy, Phys. Rev. D 79, 115018 (2009) arXiv:0902.0170 [hep-ph]].

[11] M. Aoki, S. Kanemura, K. Tsumura and K. Yagyu, Phys. Rev. D 80, 015017 (2009) arXiv:0902.4665 [hep-ph]].

[12] S. Su and B. Thomas, Phys. Rev. D 79, 095014 (2009) arXiv:0903.0667 [hep-ph]].

[13] H. E. Logan and D. MacLennan, Phys. Rev. D 79, 115022 (2009) [arXiv:0903.2246 [hep-ph]].

[14] J. Cao, P. Wan, L. Wu and J. M. Yang, Phys. Rev. D 80, 071701 (2009) arXiv:0909.5148 [hep-ph]].

[15] M. Aoki, S. Kanemura and O. Seto, Phys. Rev. Lett. 102, 051805 (2009) arXiv:0807.0361 [hep-ph]].

[16] A. Djouadi, J. Kalinowski and M. Spira, Comput. Phys. Commun. 108, 56 (1998) arXiv:hep-ph/9704448, code available from http://people.web.psi.ch/spira/hdecay/. 
[17] M. Schmaltz and D. Tucker-Smith, Ann. Rev. Nucl. Part. Sci. 55, 229 (2005) arXiv:hep-ph/0502182.

[18] G. Aad et al. [The ATLAS Collaboration], arXiv:0901.0512 [hep-ex].

[19] G. Aarons et al. [ILC Collaboration], arXiv:0709.1893 [hep-ph].

[20] V. Barger, M. McCaskey and G. Shaughnessy, Phys. Rev. D 82, 035019 (2010) arXiv:1005.3328 [hep-ph]].

[21] J. R. Ellis, A. Ferstl and K. A. Olive, Phys. Lett. B 481, 304 (2000) arXiv:hep-ph/0001005.

[22] C. E. Aalseth et al. [CoGeNT collaboration], arXiv:1002.4703 [astro-ph.CO].

[23] E. Aprile et al. [XENON100 Collaboration], Phys. Rev. Lett. 105, 131302 (2010) arXiv:1005.0380 [astro-ph.CO]]. 